\title{
Reducing the temperature sensitivity of SOI waveguide-based biosensors
}

\author{
Kristinn B. Gylfason*a , Albert Mola Romero ${ }^{\mathrm{a}, \mathrm{b}}$, Hans Sohlström ${ }^{\mathrm{a}}$ \\ ${ }^{a}$ KTH Royal Institute of Technology, Microsystem Tech. Lab., SE-100 44 Stockholm, Sweden; \\ ${ }^{b}$ University of Barcelona, Avinguda Diagonal, 647, 08028 Barcelona, Spain
}

\begin{abstract}
Label-free photonic biosensors fabricated on silicon-on-insulator (SOI) can provide compact size, high evanescent field strength at the silicon waveguide surface, and volume fabrication potential. However, due to the large thermo optic coefficient of water-based biosamples, the sensors are temperature-sensitive. Consequently, active temperature control is usually used. However, for low cost applications, active temperature control is often not feasible.

Here, we use the opposite polarity of the thermo-optic coefficients of silicon and water to demonstrate a photonic slot waveguide with a distribution of power between sample and silicon that aims to give athermal operation in water.

Based on simulations, we made three waveguide designs close to the athermal point, and asymmetric integrated MachZehnder interferometers for their characterization. The devices were fabricated on SOI with a $220 \mathrm{~nm}$ device layer and $2 \mu \mathrm{m}$ buried oxide, by electron beam lithography of hydrogen silsesquioxane (HSQ) resist, and etching in a $\mathrm{Cl}_{2} / \mathrm{HBr} / \mathrm{O}_{2} / \mathrm{He}$ plasma. With Cargile 50350 fused silica matching oil as top cladding, the group index of the three guides varies from 1.9 to 2.8 at $1550 \mathrm{~nm}$. The temperature sensitivity of the devices varied from -70 to $-160 \mathrm{pm} / \mathrm{K}$ under the same conditions. A temperature sensitivity of $-2 \mathrm{pm} / \mathrm{K}$ is projected with water as top cladding.
\end{abstract}

Keywords: Slot waveguide, biosensor, athermal, temperature sensitivity

\section{INTRODUCTION}

Lab-on-Chip biosensors offer real-time measurements, small size, portability and low sample consumption. This can make diagnostics faster and easier for the patient and the doctor. In addition, Lab-on-Chip biosensors can be used everywhere, at the doctor's office or at home, without the need of a specialized laboratory for the analysis.

Optical Lab-on-Chip biosensors integrate fluidic and photonic functions in a compact form. However, since refractive index sensors are highly temperature-dependent, external heaters or coolers are often employed to stabilize the chip temperature. The need for active temperature control adds cost, size and complexity to the sensor.

Several ways to athermalize waveguide devices, so that they can be used without active temperature control, have previously been shown $[1,2,3]$. Here, we will show that using slot-waveguides and taking advantage of the opposite polarity of the Thermo-Optic Coefficients (TOCs) of the core and the liquid top cladding, an athermal waveguide can be designed without sacrificing the high refractive index sensitivity achievable with slot waveguides [4].

Optical confinement in a slot waveguide was first introduced in [5] and experimentally verified in [6] by Almeida et al. in 2004. Slot-waveguides allow us to confine light in a low refractive index material such as water. Another advantage of this type of waveguide is the ability to design athermal waveguide biosensors by choosing proper slot and rails dimensions and suitable materials. Table 1 lists some reports on athermal slot-waveguides.

In 2008, Lee et al. showed that the temperature dependence of a silicon waveguide can be controlled using a slotwaveguide structure filled with a polymer (WIR30-490) upper cladding [7]. The intended application was temperatureinsensitive silicon ring add-drop filters, arrayed waveguide gratings (AWGs) and ring modulators.

In 2009, Wang et al. proposed an athermal silicon AWG using a polymer filled slot structure [8]. The same year, Zhou et al. experimentally demonstrated a reduction in thermal sensitivity of slotted silicon microring resonators by using polymethyl methacrylate (PMMA) as an upper cladding $[9,10]$.

*kristinn.gylfason@ee.kth.se; phone +46 879092 31; fax +46 81008 58; www.ee.kth.se/mst

Silicon Photonics and Photonic Integrated Circuits III, edited by Laurent Vivien, Seppo K. Honkanen, Lorenzo Pavesi, Stefano Pelli, Proc. of SPIE Vol. 8431, 84310F · @ 2012 SPIE · CCC code: 0277-786X/12/\$18 · doi: 10.1117/12.922263 
In 2010, Huang et al. presented a temperature-independent Mach-Zehnder interferometer (MZI) based optical filter. The design was verified via a two-dimensional finite difference time domain simulation [11]. In the same year, we presented an integrated slot-waveguide refractive index biosensor array fabricated in silicon nitride on silica. We suggested the possibility of athermal slot-waveguide refractive index sensors, tuning the slot width to balance the fraction of light propagating in the liquid sample [12]. Atsumi et al. proposed an athermal silicon slot-waveguide ring resonator embedded with benzocyclobutene (BCB) for large scale integration (LSI) chip applications [13].

Table 1. Reports on athermal slot-waveguides

\begin{tabular}{llcl}
\hline Year & Description & Temperature sensitivity $[\mathrm{pm} / \mathrm{K}]$ & Ref \\
\hline 2008 & Ring resonator filter & -2 & {$[7]$} \\
2009 & Arrayed waveguide grating (AWG) & 0.14 & {$[8]$} \\
2009 & Ring resonator filter & $52 / 27$ & {$[9,10]$} \\
2010 & Mach-Zehnder interferometer & 1 & {$[11]$} \\
2010 & Ring resonator sensor array & -16.6 & {$[12]$} \\
2010 & Ring resonator filter & -0.6 & {$[13]$} \\
\hline
\end{tabular}

Here we use simulations to show that silicon slot waveguides with an upper cladding of water can be made athermal (section 2). We also describe the design and fabrication of Mach-Zhender waveguide interferometers to characterize the slot waveguides (section 3 and 4). We then give measurement results that show that the waveguide design works as expected and should give a TOC close to zero with water (section 5), before we conclude (section 6).

\section{ATHERMAL SLOT-WAVEGUIDE}

\subsection{Athermal slot-waveguide simulation}

Water has a negative TOC of $\kappa_{\mathrm{H} 2 \mathrm{O}}=-1.1 \cdot 10^{-4} \mathrm{RIU} / \mathrm{K}$ [14], while silicon and silica have positive values of $\kappa_{\mathrm{Si}}=2.4 \cdot 10^{-4} \mathrm{RIU} / \mathrm{K}$ [8] and $\kappa_{\mathrm{SiO} 2}=0.1 \cdot 10^{-4} \mathrm{RIU} / \mathrm{K}$ [15], respectively. Thus, by balancing the fraction of light propagating in each material, an athermal waveguide can be designed. The temperature dependence of the effective waveguide refractive index can be approximated using the optical power fraction propagating in each material $\left(P_{\mathrm{H} 2 \mathrm{O}}, P_{\mathrm{Si}}\right.$ and $P_{\mathrm{SiO} 2}$ ) multiplied by the respective TOCs

$$
\frac{\partial n_{e f f}}{\partial T} \approx \kappa_{\mathrm{H}_{2} \mathrm{O}} \cdot P_{\mathrm{H}_{2} \mathrm{O}}+\kappa_{\mathrm{Si}} \cdot P_{\mathrm{Si}}+\kappa_{\mathrm{SiO}_{2}} \cdot P_{\mathrm{SiO}_{2}}
$$

Figure 1 shows a cross section of a slot-waveguide, indicating the sign of the TOCs of the different materials.

Using a finite element mode solver (COMSOL Multiphysics ${ }^{\mathrm{TM}}$ ), the effect of the geometrical parameters of the athermal slot-waveguide cross section can be simulated. We consider only the $\mathrm{TE}_{0}$ mode, since this is the most interesting mode for sensing. Sweeping the slot width $w_{\text {slot }}$ and the rail width $w_{\text {rail }}$, equation (1) has been evaluated. The waveguide height was chosen $220 \mathrm{~nm}$ to conform to an established industry standard, used by e.g. the ePIXfab silicon foundry [16].

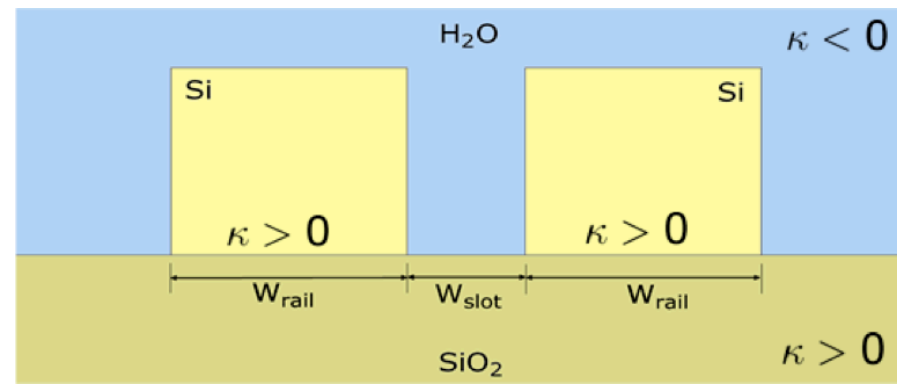

Figure 1. A cross section of the slot-waveguide indicating the sign of the TOCs of the materials 
The results are shown in Figure 2. We see that as the slot width increases, larger rail width is needed to achieve athermal operation. A $120 \mathrm{~nm}$ slot width and $215 \mathrm{~nm}$ rail width slot waveguide (B) gives a TOC close to zero. We select these parameters for the athermal guide. In order to have more experimental points we also chose to fabricate two more slotwaveguides with different rail dimensions: one with $180 \mathrm{~nm}$ rail width (A), and one with $240 \mathrm{~nm}$ rail width (C), cf. Table 2. The slot width is $120 \mathrm{~nm}$ in all the cases.

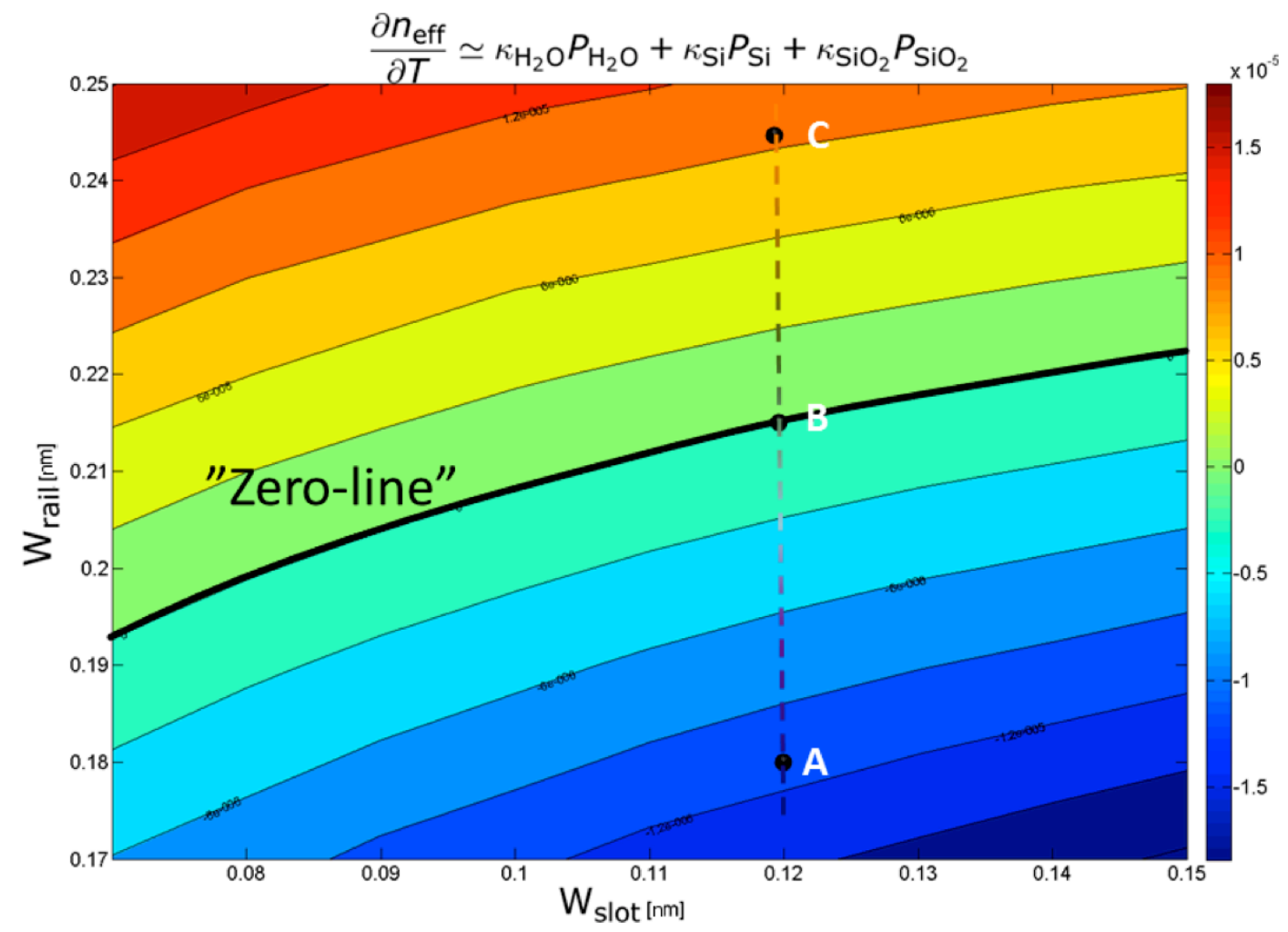

Figure 2. A contour plot of the $n_{\text {eff }}$ temperature dependence as a function of the rail width and the slot width showing the "zero-line" of athermal operation. The dashed line shows one transition from a positive TOC to a negative TOC, and the corresponding slot waveguides chosen for fabrication: A, a slot-waveguide with $180 \mathrm{~nm}$ rail width, with a negative TOC. B, a slot-waveguide with $215 \mathrm{~nm}$ rail width, on the "zero line". C, a slot-waveguide with $240 \mathrm{~nm}$ rail width, with a positive TOC. The slot width has been fixed at $120 \mathrm{~nm}$ and the rail height at $220 \mathrm{~nm}$ in all cases.

For the evaluation of the waveguides, their group index $n_{\mathrm{g}}$ is a key property. Using the values of $\partial n_{\text {eff }} / \partial \lambda$ obtained from simulations of $n_{\text {eff }}$ at different wavelengths and the formula

$$
n_{\mathrm{g}}=n_{\mathrm{eff}}-\frac{\partial n_{\mathrm{eff}}}{\partial \lambda}
$$

we calculated the expected values for guide group indices, cf. Table 2.

Table 2. Simulated $\partial n_{\mathrm{eff}} / \partial T$ and $n_{\mathrm{g}}$ values for the guides included in the study. $n_{\mathrm{g}}$ is given for water cladding and for cladding with the index matching oil used in our experiments.

\begin{tabular}{cccc}
\hline Slot-waveguide type & $\partial n_{\mathrm{eff}} / \partial T$ & $\begin{array}{c}n_{\mathrm{g}} \text { with water } \\
n_{\mathrm{H} 2 \mathrm{O}}=1.32\end{array}$ & $\begin{array}{c}n_{\mathrm{g}} \text { with index matching oil } \\
n_{\mathrm{oil}}=1.449\end{array}$ \\
\hline Rail $180 \mathrm{~nm}$ & $-1.0954 \cdot 10^{-5} \mathrm{RIU} / \mathrm{K}$ & 1.95 & 2.30 \\
Rail $215 \mathrm{~nm}$ & $-4.8808 \cdot 10^{-8} \mathrm{RIU} / \mathrm{K}$ & 2.52 & 2.71 \\
Rail $240 \mathrm{~nm}$ & $+7.8804 \cdot 10^{-6} \mathrm{RIU} / \mathrm{K}$ & 2.88 & 3.00 \\
\hline
\end{tabular}




\section{ASYMMETRIC MACH-ZEHNDER INTERFEROMETER DESIGN}

\subsection{MZI design}

In order to measure the effective refractive index temperature dependence of the slot waveguide, we use asymmetric MZIs, as shown in Figure 3. The input sections, the splitter, the bends, the combiner and the output sections are made with conventional $450 \mathrm{~nm}$ wide strip waveguides. Each arm contains two strip-to-slot converters, two straight slot waveguides (each of length $L_{\mathrm{i}} / 2, i=1,2$ ) and two slot-to-strip converters. The designs of the two arms are identical except for the length, $L_{\mathrm{i}}$ of the slot waveguides.

The radii of the input and output bends of the arms of the MZI were made $90 \mu \mathrm{m}$, which is large enough to give a $90 \mu \mathrm{m}$ margin between the MMI splitter and the arms of the MZI, and between the arms and the directional coupler, for the location of a fluidic channel for the evaluation with water. At the same time it is small enough to allow us to write the whole design in a single $800 \mu \mathrm{m} \times 800 \mu \mathrm{m}$ write field on the Raith-150 electron beam writer used.

If there is no asymmetry, the optical paths of light in both arms of the interferometer are equal. That means that no phase difference will arise with changing temperature and no interference is produced.

If the MZI is unbalanced by $\Delta L=L_{1}-L_{2}$, the Free Spectral Range (FSR), $\Delta \lambda$, i.e. the spacing between two consecutive wavelengths with constructive or destructive interference can be approximated [17] as

$$
\Delta \lambda=\frac{\lambda^{2}}{n_{\mathrm{g}} \Delta L}
$$

and the wavelength temperature sensitivity of the MZI as

$$
\frac{\partial \lambda}{\partial T}=\frac{\lambda}{n_{\mathrm{g}}} \cdot \frac{\partial n_{\text {eff }}}{\partial T}
$$
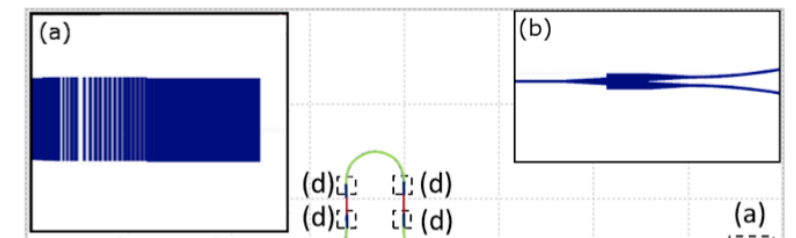

(d) it: iti (d)

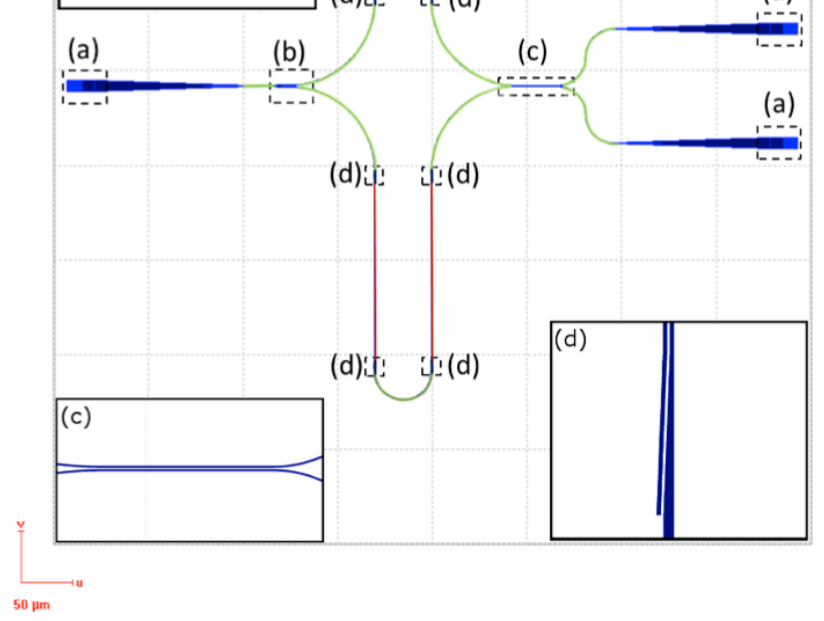

Figure 3. A top view of the layout of the asymmetric Mach-Zehnder interferometer (MZI): Light is coupled into the left surface grating coupler (a) and split by the MMI splitter (b) into the two arms of the MZI. Light is coupled to the slot waveguide trough the strip-slot converters (d). The beams of the two arms of the MZI interfere in the directional coupler (c) and are coupled out through the output surface couplers. The slot-waveguide regions are between the stripslot converters. 
To chose a $\Delta L$ for the evaluation we note that if the asymmetry is large, the resulting visibility of the spectra may be low, due to the propagation loss of the waveguides. Furthermore, a large asymmetry leads to a large device footprint. On the other hand, a small asymmetry will cause a large FSR, making it impossible to see several periods of the interference patterns in a limited wavelength tuning range. With a wavelength tuning range of $120 \mathrm{~nm}$, and a minimum step size of $1 \mathrm{pm}$, we can use a $1 \mathrm{~nm}$ FSR, giving many measurable points in one period.

From (3) we see that an FSR of $1 \mathrm{~nm}$, a wavelength of $1550 \mathrm{~nm}$ and a group index of 2.52 gives $\Delta L=953 \mu \mathrm{m}$. Based on this we designed an MZI with $\Delta L=900 \mu \mathrm{m}$. To improve the visibility and to allow us to make a good group index evaluation, also devices with $\Delta L=660 \mu \mathrm{m}$ and $\Delta L=330 \mu \mathrm{m}$ were designed.

The silicon slot-waveguides have an estimated propagation loss of $L_{\mathrm{dB}}=10 \mathrm{~dB} / \mathrm{cm}$ [18]. The beam intensity ratio $r$ only depends on the difference in loss between the two arms of the MZI. Table 3 shows estimations of the loss difference due to the asymmetry, and the resulting beam intensity ratio and visibility, for each $\Delta L$.

Table 3. Projected loss, beam intensity ratio and visibility for the different $\Delta L$.

\begin{tabular}{cccc}
\hline$\Delta L[\mu \mathrm{m}]$ & $L_{\mathrm{dB}} \Delta L[\mathrm{~dB}]$ & $r$ & $V$ \\
\hline 900 & 0.90 & 0.41 & 0.91 \\
660 & 0.66 & 0.52 & 0.95 \\
330 & 0.33 & 0.72 & 0.99 \\
\hline
\end{tabular}

\subsection{Wavelength temperature sensitivities of the MZI}

From equation (4), the simulated group index values for the slot-waveguides operating in water and their TOCs, the expected temperature sensitivities $\partial \lambda / \partial T$ can be calculated. Table 4 shows the values for the different slot-waveguide designs, operating in water, evaluated at a wavelength of $1550 \mathrm{~nm}$.

Table 4. Expected temperature sensitivities for the different slot-waveguides at $1550 \mathrm{~nm}$ operating in water.

\begin{tabular}{cc}
\hline Slot-waveguide type & Temperature sensitivity \\
\hline Rail $180 \mathrm{~nm}$ & $-8.69 \mathrm{pm} / \mathrm{K}$ \\
Rail $215 \mathrm{~nm}$ & $-0.03 \mathrm{pm} / \mathrm{K}$ \\
Rail $240 \mathrm{~nm}$ & $+4.24 \mathrm{pm} / \mathrm{K}$ \\
\hline
\end{tabular}

\subsection{Multi-mode interference splitter}

For the MZI we need a waveguide splitter and a combiner. For the splitting, we have chosen a multi-mode interference (MMI) splitter [19]. Other types of power splitters and combiners are shown in the literature, such as Y-junctions [20] and directional couplers [21]. Compact low loss MMI couplers have been designed in [22].

Using the effective index method and 2D simulations using COMSOL Multiphysics ${ }^{\mathrm{TM}}$, we investigated different MMI splitter designs. The optical power ratio $(O P R)$, which is the ratio of the input optical power that is coupled to the two outputs, gives a measure of the loss in the structure.

$$
O P R=\frac{P_{\text {out }}}{P_{\text {in }}}
$$

The contrast of the interference patterns emerging in the input and output guides is a measure of the amount of reflections in the MMI. The reflection parameter, $R$, is defined as

$$
R=\frac{\left|E_{\max }\right|-\left|E_{\min }\right|}{\left|E_{\max }\right|},
$$

where $E_{\max }$ and $E_{\min }$ are the maximum and minimum field strengths, respectively. If $R \rightarrow 0$, the reflections are minimal, and if $R \rightarrow 1$, the reflections are maximal. The reflection parameter has been evaluated along sections of the input and 
output waveguides in the simulation results. $R_{\text {in }}$ is the reflection evaluation along the input, and $R_{\text {out }}$ the reflection evaluation along one of the outputs.

Three MMI splitter designs were studied: an MMI splitter without tapers (Type I), an MMI splitter with identical linear tapers at the input and at the output, (Type II), and an MMI splitter combining linear tapers and exponential tapers (Type III) [23]. These structures have been simulated and the $O P R$ and $R$ quantified. Figure 4.3 shows the different structures.


Figure 4. The three MMI splitters studied: (a) Type I MMI splitter, with no tapers. (b) Type II MMI splitter, with equal linear tapers at the input and at the outputs. (c) Type III MMI splitter, with linear tapers at the outputs and an exponential taper at the input.

The distance between the output arms is chosen to $1.4 \mu \mathrm{m}$ in all cases. The design parameters are then the width $W$ and the length $l$ of the MMI splitter cavity, the length of the input and output tapers ( $\left.L_{\text {in }}, L_{\text {out }}\right)$, and the angle of the input and output tapers $\left(\theta_{\text {in }}, \theta_{\text {out }}\right)$.

Table 5 shows the optimal design parameters for each splitter type in terms of OPR and reflections. Type I gives most reflections and least OPR. Type II gives the best results in terms of the optical power coupling, and type III the lowest reflections. After comparing the different types of MMI splitters, we find that type II is the one that give the best overall optical performance. The total length of the device is only $21 \mu \mathrm{m}$.

Table 5. The optimal design parameters and the reflection (R) and optical power ratio (OPR) evaluation of the different MMI splitters studied. Type I is the MMI splitter with no tapers, type II the MMI splitter with equal linear tapers at the input and at the outputs, and type III, is the MMI splitter with linear tapers at the output and exponential at the input.

\begin{tabular}{cccccccccc}
\hline Type & $W[\mu \mathrm{m}]$ & $l[\mu \mathrm{m}]$ & $L_{\text {in }}[\mu \mathrm{m}]$ & $L_{\text {out }}[\mu \mathrm{m}]$ & $\theta_{\text {in }}\left[^{\circ}\right]$ & $\theta_{\text {out }}\left[{ }^{\circ}\right]$ & $R_{\text {in }}$ & $R_{\text {out }}$ & $O P R$ \\
\hline I & 2.7 & 6.9 & 0 & 0 & 0 & 0 & 0.14 & 0.14 & 0.75 \\
II & 2.7 & 6.9 & 7 & 7 & 2.2 & 2.2 & 0.09 & 0.11 & 0.98 \\
III & 3.0 & 3.0 & 7 & 2 & - & 3 & 0.08 & 0.10 & 0.96 \\
\hline
\end{tabular}

\subsection{Combiner}

The purpose of the combiner is to combine the beams of the two arms of the MZI. Ideally, when the beams are in phase, the total optical power propagating in the interferometer should appear at the output. When the beams are out of phase, there should not be any light at the output. In reality, there are reflections and losses that deteriorate these ideal values.

We simulated the use of MMI combiners with the same geometric designs as for the MMI splitters (Figure 4), but interchanging inputs and outputs. However, reflections are visible in all types, particularly when light is out of phase. This makes the MMI coupler less suitable as combiner for high index contrast waveguides. 
As combiners, directional couplers give a better performance, although they are less compact.. The design parameters are the width of the waveguides $w_{\mathrm{g}}$, the arm separation $d$ and the coupling length $L_{\mathrm{c}}$, Figure 5. A characteristic of directional couplers is that the coupling length increases exponentially with the arm separation [24]. This means that to have a compact design, a small arm separation is needed. For ease of fabrication, a directional coupler with a $400 \mathrm{~nm}$ arm separation is chosen as a compromise.

After the evaluation of different directional couplers, we decide to take for the final design the directional coupler formed from $w_{\mathrm{g}}=450 \mathrm{~nm}$ wide waveguides, separated by $d=400 \mathrm{~nm}$, with a coupling length of $L \mathrm{C}=42.8 \mu \mathrm{m}$.

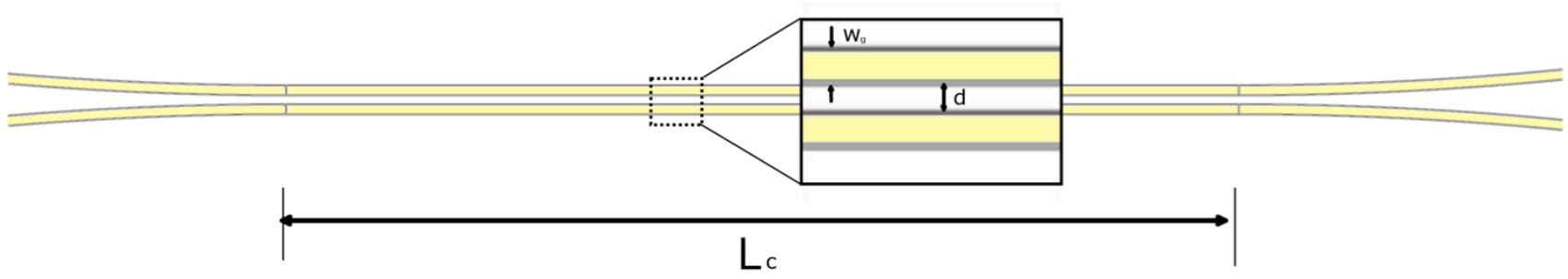

Figure 5. A schematic of the directional coupler, with an enlargement showing the coupling gap.

\subsection{Strip to slot mode converter}

Strip to slot mode converters are needed to connect these two types of waveguides without significant losses. Different designs have been proposed [25,26]. In the first (Figure 6 (a)), a strip-waveguide is tapered to form one rail of the slotwaveguide and the second rail is funneled from the side of the strip-waveguide. In the second (Figure 6 (b)), the stripwaveguide is tapered symmetrically forming a tip, and the rails of the slot-waveguide are funneled in from both sides.

A drawback of case (b) is the tip at the end of the strip waveguide taper. Reports shows that for a $50 \mathrm{~nm}$ wide tip there is an efficiency drop from $99.9 \%$ to around $96.0 \%$ [26]. This fact makes design (b) more critical in terms of fabrication.

Bigger lossless slot-strip mode converters have been reported in [27], but for compactness and ease of fabrication, we chose the design shown in Figure 6(a) for the final design with a $7 \mu \mathrm{m}$ taper length.

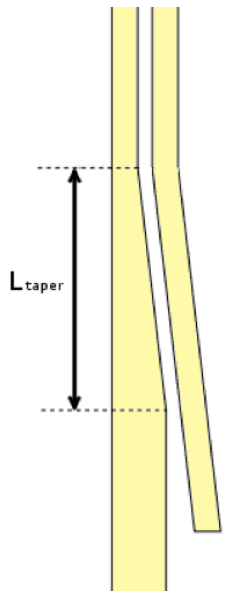

(a)

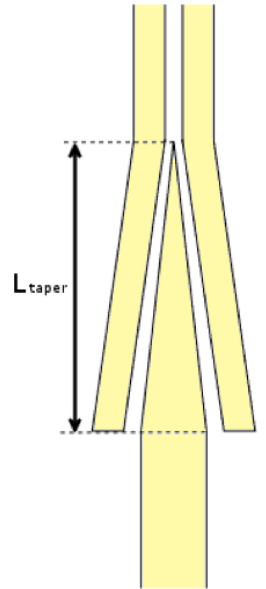

(b)

Figure 6. Schematic diagrams of the two proposed strip-to-slot couplers.

\subsection{Grating couplers}

Optical fibers are used to transport light from an external source to the optical chip. There are two strategies for coupling light into the on-chip waveguides. The first one is to focus the light into the waveguide end-face at the chip edge [28]. The coupling efficiency can be improved with lensed fibers, vertical tapers [28], or graded refractive index lenses [29]. However, in all cases, it is required to polish the input waveguide end-face, making this option inconvenient and costly. 
The second option is to use surface grating couplers. The width of the grating guide is chosen $12 \mu \mathrm{m}$ to approximately match the fiber mode diameter [30]. The grating is then tapered into the single-mode waveguide. This coupling strategy allows higher coupling efficiency and avoids the need for polishing the waveguide end-face.

Different types of grating couplers are presented in the literature. Coupling efficiencies of $34 \%$ for a periodic grating were reported in [31]. With a surface grating made of fully etched nano-holes, the coupling efficiency achieved was $42 \%$ [32]. Another strategy to improve the coupling efficiency is by using Bragg mirror reflectors below the gratings to reduce the loss to the substrate [33]. In this case, a coupling efficiency of $69.5 \%$ was achieved. Recently, we proposed a trough-etched SOI grating with fill factor apodization with a simulated maximum coupling efficiency of $72 \%$ [34]. The gratings were apodized by varying the width of each bar and gap individually using a genetic algorithm [35] giving low back reflections into the silicon-waveguide, the possibility to use standard SOI wafers, and a single lithography for the fabrication. The gratings used in the present work, Figure 7, are based on this design.



Figure 7. The layout of the gratings and tapers used in this work.

\section{FABRICATION}

\subsection{Fabrication sequence}

The optical chip was micro-fabricated in SOI with a $220 \mathrm{~nm}$ silicon device layer and a $2 \mu \mathrm{m}$ silica layer.

We employed a negative electron beam resist (XR-1541-2\% hydrogen silsesquioxane (HSQ), Dow Corning Corporation [36]). A negative electron beam resist is well suited for electron beam patterning of narrow waveguides, since only a small fraction of the surface has to be exposed. The resist was dispensed and spun in two different steps, $5 \mathrm{~s}$ spread at $500 \mathrm{rpm}$ and $30 \mathrm{~s}$ spin at $2000 \mathrm{rpm}$.

After spin-coating, the chips were baked on a hotplate in two steps $\left(2 \mathrm{~min}\right.$ at $150^{\circ} \mathrm{C}$ and $2 \mathrm{~min}$ at $\left.220^{\circ} \mathrm{C}\right)$. This soft bake evaporates the solvent and improves the adhesion of the resist to the wafer.

The patterns were exposed with a Raith-150 electron beam writer (Raith GmbH, Germany), with a $10 \mu \mathrm{m}$ aperture at an acceleration voltage of $25 \mathrm{kV}$. The chip was developed in ma-D 532s developer (TMAH) for 2 minutes. Finally, a $400^{\circ} \mathrm{C}$ hard bake on a hot plate during 40 minutes was made to make the resist layer more resistant to the plasma etching.

A Precision 5000 Mark II (Applied Materials, United States) was used for the silicon dry etching in a $\mathrm{Cl}_{2} / \mathrm{HBr} / \mathrm{He} / \mathrm{O}_{2}$ plasma. Finally, the resist was stripped in HF 5\% during $20 \mathrm{~s}$. 


\subsection{Inspection of the optical chip}

After the fabrication the designs were checked with scanning electron microscopy (SEM). Figure 8 shows an optical image of the $900 \mu \mathrm{m}$ asymmetry design with zoom-in SEM pictures of the MMI, the directional coupler, a surface grating and a strip-slot converter.

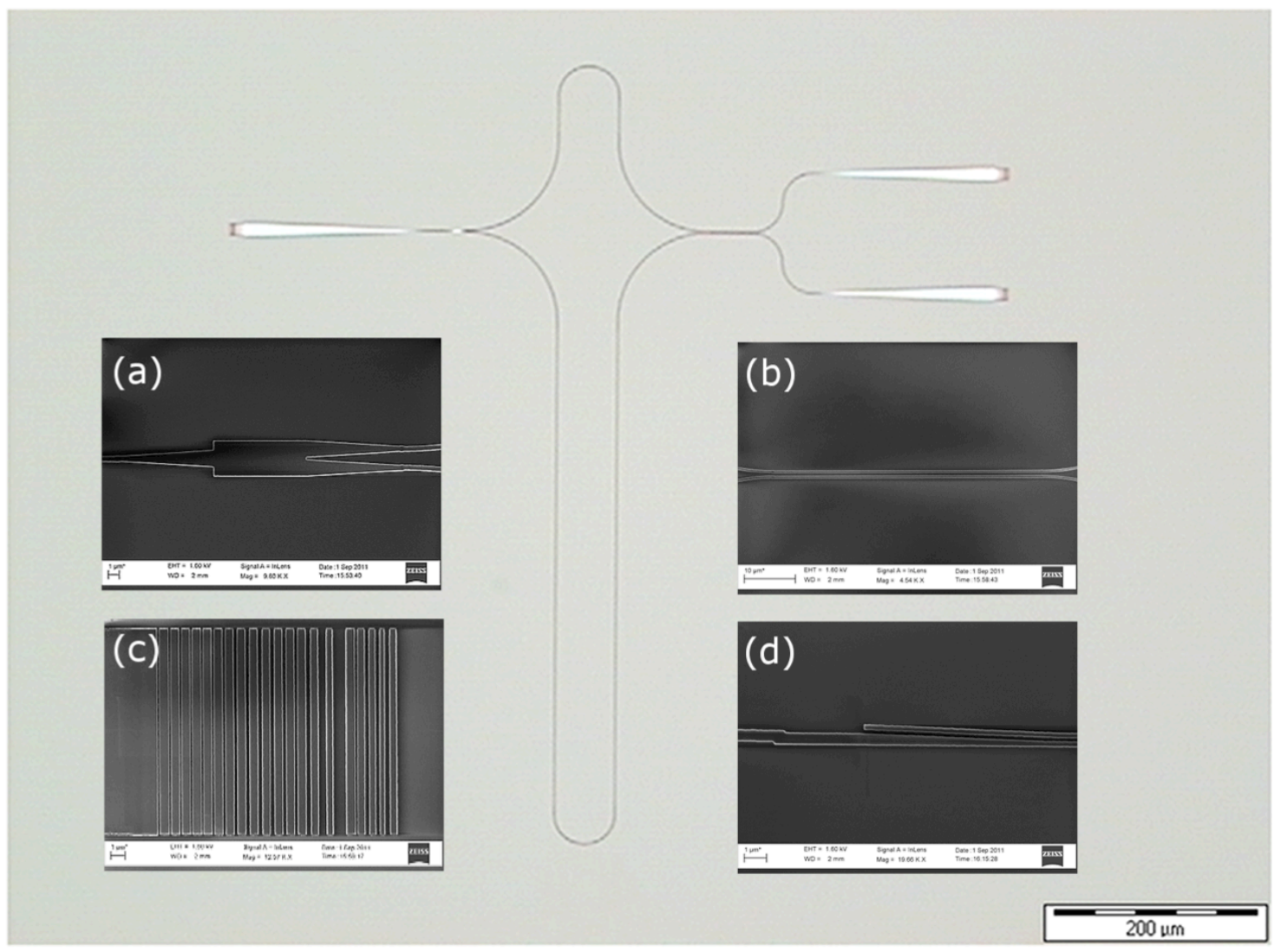

Figure 8. An optical image of the $900 \mu \mathrm{m}$ asymmetry MZI. The insets show SEM images of (a) the surface grating, (b) the directional coupler, (c) the MMI, and (d) the strip-slot converter

Table 6. Designed and measured slot-waveguide dimensions.

\begin{tabular}{lllllll}
\hline \multicolumn{3}{c}{ Designed } & \multicolumn{3}{c}{ Fabricated } \\
\hline Asymmetry $[\mu \mathrm{m}]$ & $w_{\mathrm{r} 1}[\mathrm{~nm}]$ & $w_{\mathrm{s}}[\mathrm{nm}]$ & $w_{2}[\mathrm{~nm}]$ & $w_{\mathrm{r} 1}[\mathrm{~nm}]$ & $w_{\mathrm{s}}[\mathrm{nm}]$ & $w_{\mathrm{r} 2}[\mathrm{~nm}]$ \\
\hline 0 & 180 & 120 & 180 & 114 & 169 & 119 \\
& 215 & 120 & 215 & 161 & 174 & 172 \\
& 240 & 120 & 240 & 189 & 169 & 211 \\
\hline 330 & 180 & 120 & 180 & 140 & 144 & 150 \\
& 215 & 120 & 215 & 170 & 155 & 182 \\
& 240 & 120 & 240 & 204 & 155 & 217 \\
660 & 180 & 120 & 180 & 144 & 142 & 145 \\
& 215 & 120 & 215 & 169 & 157 & 188 \\
& 240 & 120 & 240 & 207 & 162 & 207 \\
\hline 900 & 180 & 120 & 180 & 139 & 156 & 149 \\
& 215 & 120 & 215 & 169 & 155 & 189 \\
& 240 & 120 & 240 & 202 & 168 & 213 \\
\hline
\end{tabular}


After the fabrication, calibrated SEM linescans were made with the Raith 150 to evaluate the dimensions of the structures. Table 6 shows the rail $\left(w_{\mathrm{r} 1}, w_{\mathrm{r} 2}\right)$ and slot $\left(w_{\text {slot }}\right)$ dimensions of the designed and fabricated structures. The measured widths of the rails are smaller than the designed ones, and consequently the slot widths are wider than designed. This discrepancy is mainly due to an imperfect dose setup and the fact that our measures the width at the top, ignoring and y sidewall slope.

Electron beam lithography provides a high resolution, allowing the patterning of very narrow structures. However, the proximity effect may cause problems when patterning closely placed structures: when exposing one structure, electron back scattering can lead to an undesirable exposure in adjacent regions. There are mainly three strategies to reduce the proximity effect: dose modification, shape modification and background correction exposure [37]. The dose correction is based on a pixel per pixel dose adjustment, using a sum of two Gaussian distributions [38].

Figure 9 shows SEM pictures of the fabricated gratings with and without proximity dose correction. Without correction, some gaps are open only at the top and bottom of the gratings, and not in the middle. In Figure 9 (b) the gaps are clearly open and the pins are straight. A drawback of this correction is that the exposure time increases significantly, increasing the risk for position drift between the exposed objects, causing offsets. Such an offset can be seen in Figure 8, inset (d).


Figure 9. SEM picture of the grating without proximity correction applied (a) and with proximity correction applied (b).

\section{CHARACTERIZATION}

\subsection{Optical setup}

The main purpose of the characterization is to evaluate the temperature sensitivities of the different slot-waveguides operating in water. For this aim, a fluidics layer has to be fabricated and attached to the optical chip. However, a first characterization of the optical chip was made using fused silica matching oil as upper cladding. This was done to evaluate the optical performance of the structures, to check the possibility of coupling light in and out of the chip, to evaluate the visibility of the interference pattern and to evaluate the group index of the different slot-waveguides.

An SMF-28 single mode fiber [30] was cleaved and the two tips arranged with a $10^{\circ}$ angle to the surface normal. Refractive index matching oil (Cargille 50350, [39]) was used as a top cladding. The matching oil has a refractive index of 1.449 at room temperature and $1550 \mathrm{~nm}$ wavelength, and a low viscosity, permitting filling of the grating gaps.

An Agilent 8164A tunable laser was used in combination with an Agilent 86082A Wavelength Domain Component Analyzer, to measure the spectrum of the fabricated structures. The polarization in the input fiber was optimized for TE coupling with a fiber polarization controller FPC560 (Thorlabs). 


\subsection{Results and discussion}

In all, 9 spectra were obtained from the different asymmetric MZIs operating in matching oil: one for each asymmetry $(330 \mu \mathrm{m}, 660 \mu \mathrm{m}$ and $900 \mu \mathrm{m})$ and each slot-waveguide type (180 nm rail, $215 \mathrm{~nm}$ rail and $240 \mathrm{~nm}$ rail width). Figure 10 shows transmission spectra for the MZI structure with $900 \mu \mathrm{m}$ asymmetry and a $215 \mathrm{~nm}$ rail width slot-waveguide.
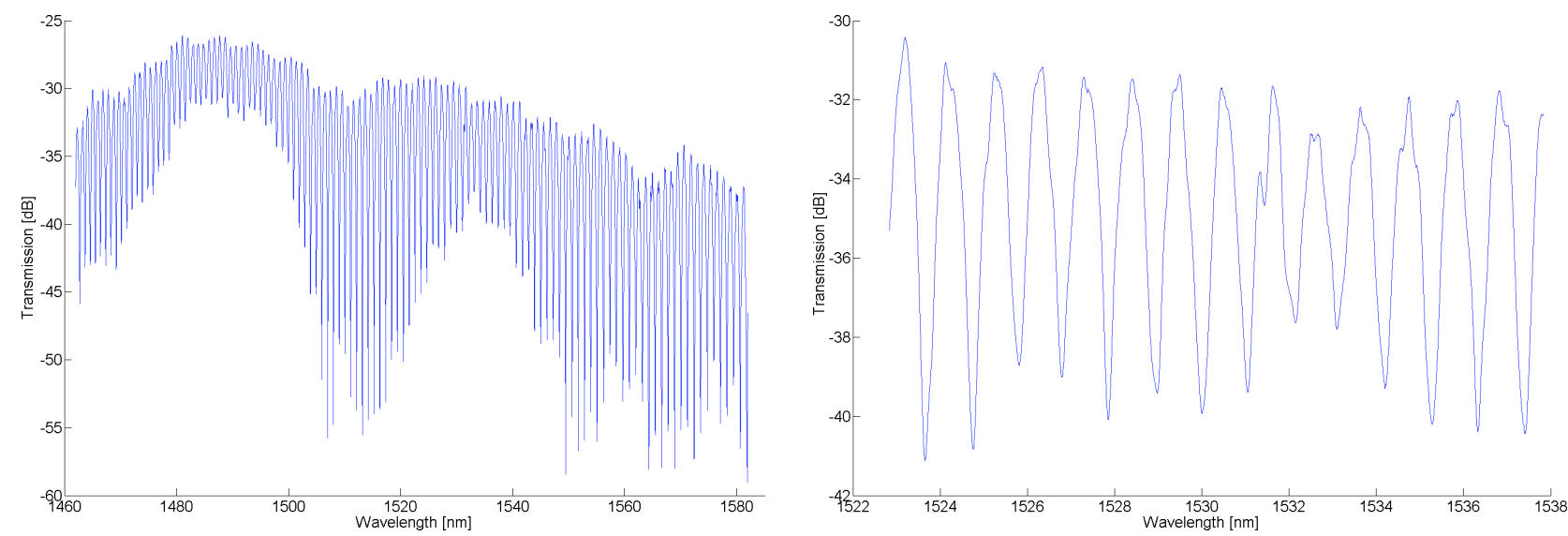

Figure 10. Transmission trough the $900 \mu \mathrm{m}$ asymmetry design Mach-Zehnder interferometer with a rail width of $215 \mathrm{~nm}$ operating in index matching oil: $120 \mathrm{~nm}$ span and $15 \mathrm{~nm}$ span.

For all the structures, interference patterns with good visibilities were obtained. The maxima and minima of all the spectra were found, and the average FSR was calculated for each structure. Table 7 shows the extinction ratio in the spectrum and the experimentally determined FSR, $\Delta \lambda(\exp )$, as well as the FSR, $\Delta \lambda(\operatorname{sim}))$, and the group index, $n_{\mathrm{g}}(\operatorname{sim})$, determined by simulations using the measured fabricated dimensions.

From equation (3) we se that $\lambda^{2} / \Delta \lambda$ depends linearly on $n_{\mathrm{g}} \Delta L$.

$$
\frac{\lambda^{2}}{\Delta \lambda}=n_{\mathrm{g}} \Delta L
$$

In Figure 11 we have fitted equation (7) to the experimental and simulated data in Table 7 to get good estimates for all the experimental and simulated group indices.

Table 7. The experimental values of extinction ratio and FSR as well as the FSR and group index from simulations using the measured fabricated dimension for the asymmetric MZIs and each slot-waveguide type.

\begin{tabular}{cccccc}
\hline Asymmetry $[\mu \mathrm{m}]$ & $w_{\mathrm{r}}[\mathrm{nm}]$ & Extinction ratio $[\mathrm{dB}]$ & $\Delta \lambda(\exp )[\mathrm{nm}]$ & $\Delta \lambda(\operatorname{sim})[\mathrm{nm}]$ & $n_{\mathrm{g}}(\operatorname{sim})$ \\
\hline 330 & 180 & -10.5 & 3.98 & 4.21 & 1.77 \\
& 215 & -29.4 & 2.95 & 3.4 & 2.09 \\
& 240 & -28.8 & 2.67 & 2.84 & 2.50 \\
660 & 180 & -8.7 & 1.74 & 2.00 & 1.77 \\
& 215 & -26.5 & 1.50 & 1.68 & 2.12 \\
& 240 & -10.4 & 1.26 & 1.46 & 2.42 \\
\hline 900 & 180 & -6.9 & 1.41 & 1.49 & 1.75 \\
& 215 & -25.5 & 1.06 & 1.24 & 2.11 \\
& 240 & -9.9 & 0.96 & 1.13 & 2.42 \\
\hline
\end{tabular}

Table 8 summarizes all the group index values: the estimation from the simulations made with the designed dimensions of the slot-waveguides, the values from simulations using the measured fabricated dimensions and the values obtained from the fitting to the experimental data. 




Figure 11. Experimental data (solid lines) and simulated from measured dimesions (dashed lines) with the corresponding fits of equation (8). The points with asterisk shape $(*)$ represent the slots-waveguides designed with $180 \mathrm{~nm}$ rail width, the points with circular shape (o) represent the slots-waveguides designed with a rail width of $215 \mathrm{~nm}$, and the points with triangular shape $(\nabla)$, the slots-waveguides designed with $240 \mathrm{~nm}$ rail widths.

The experimental values are between the simulated ones with the designed dimensions and those simulated with the measured dimensions. The values from the simulations with the designed dimensions are larger since an overestimation of the rail width is made. Since the fabricated slot-waveguides have narrower rails and wider slots than designed, the amount of light propagating in silicon is reduced, and consequently, the group index of the waveguides. On the other hand, the obtained values from the simulations with the measured values are underestimations, due to the fact that the rails are wider at the bottom than at the top where the rail widths were measured.

Table 8. Group index obtained from the simulations and the experimental data for the different slot waveguide geometries using fused silica as a top cladding.

\begin{tabular}{lccc}
\hline Slot-waveguide type & Rail $180 \mathrm{~nm}$ & Rail 215 $\mathrm{nm}$ & Rail 240 $\mathrm{nm}$ \\
\hline Simulated $n_{\mathrm{g}}$ (Designed dimensions) & 2.30 & 2.71 & 3.00 \\
Experimental $n_{\mathrm{g}}$ & 1.94 & 2.44 & 2.86 \\
Simulated $n_{\mathrm{g}}$ (Measured dimensions) & 1.75 & 2.11 & 2.42 \\
\hline
\end{tabular}

As a first step, a preliminary test of the temperature sensitivity of the slot-waveguides operating in silica matching oil was made. This gives us a first idea of the thermal performance of the different structures. Taking into account the fact that the matching oil has a negative TOC of $-3.86 \cdot 10^{-4} \mathrm{RIU} / \mathrm{K}$ [39], a corresponding level of temperature compensation should be evident.

The temperature control setup consists of a power resistor MP915 (Caddock Electronics, Inc.) attached to an aluminum plate. The temperature control was designed to operate at four different temperatures: $30^{\circ} \mathrm{C}, 34^{\circ} \mathrm{C}, 38^{\circ} \mathrm{C}$ and $43^{\circ} \mathrm{C}$.

Figure 12 shows the transmission spectra of the $330 \mu \mathrm{m}$ asymmetry MZI, with a designed rail width of $240 \mathrm{~nm}$, for two different temperatures, $30^{\circ} \mathrm{C}$ and $34^{\circ} \mathrm{C}$. Similar spectra was also recorded for the MZI with $660 \mu \mathrm{m}$ asymmetry. From these spectra, the experimental wavelength shift $\partial \lambda / \partial \mathrm{T}$ with the temperature was determined to $-70 \mathrm{pm} / \mathrm{K}$ from the $330 \mu \mathrm{m}$ asymmetry MZI and $-160 \mathrm{pm} / \mathrm{K}$ for the MZI with $660 \mu \mathrm{m}$ asymmetry. 




Figure 12. The transmission spectrum of the $330 \mu \mathrm{m}$ asymmetry MZI, with a designed rail width of $240 \mathrm{~nm}$ for two different temperatures, $30^{\circ} \mathrm{C}$ and $34^{\circ} \mathrm{C}$ with index matching oil cladding.

To determine the theoretical values from equation (4), the TOC of the slot-waveguide of the MZIs with $330 \mu \mathrm{m}$ and $660 \mu \mathrm{m}$ asymmetry with the measured dimensions shown in Table 6 , and with oil as a top cladding has been calculated based on the simulations. For these slot-waveguides, the group index as determined from simulations based on measured dimension is $n_{\mathrm{g}}=2.50(330 \mu \mathrm{m}$ asymmetry) and $2.42(660 \mu \mathrm{m}$ asymmetry), as has been shown in Table 7. Using simulation values and taking into account the silicon thermal expansion, the theoretical temperature sensitivity for these cases is $-73 \mathrm{pm} / \mathrm{K}$ (330 $\mu \mathrm{m}$ asymmetry) and $-190 \mathrm{pm} / \mathrm{K}(660 \mu \mathrm{m}$ asymmetry). Considering the uncertainty in the geometry measurement, the fit between simulations and experiment is reasonable.

The expected temperature sensitivities by using water as a top cladding were also calculated. For this purpose, the group index and the effective refractive index at a wavelength of $1550 \mathrm{~nm}$ for all the structures, operating in water, were calculated. Table 9 shows the refractive index, the TOCs and the expected temperature sensitivities for all the fabricated structures.

Table 9. Evaluation of the group index, the effective refractive index, the TOC and the expected temperature sensitivity of all the fabricated structures operating in water.

\begin{tabular}{lccccccc}
\hline Asymmetry $[\mu \mathrm{m}]$ & $w_{\mathrm{r} 1}[\mathrm{~nm}]$ & $w_{\mathrm{s}}[\mathrm{nm}]$ & $w_{\mathrm{r} 2}[\mathrm{~nm}]$ & $n_{\mathrm{g}}$ & $n_{\mathrm{eff}}$ & TOC $[\mathrm{RIU} / \mathrm{K}]$ & Temperature sensitivity $[\mathrm{pm} / \mathrm{K}]$ \\
\hline 330 & 140 & 144 & 150 & 1.57 & 1,44 & $-3.2610^{-5}$ & -28.44 \\
& 170 & 155 & 182 & 1.73 & 1,46 & $-2.5710^{-5}$ & -19.63 \\
& 204 & 155 & 217 & 2.28 & 1,54 & $-6.2510^{-6}$ & -1.53 \\
\hline 660 & 144 & 142 & 145 & 1.57 & 1,42 & $-3.2610^{-5}$ & -28.50 \\
& 169 & 157 & 188 & 1.76 & 1,46 & $-2.3810^{-5}$ & -17.61 \\
900 & 207 & 162 & 207 & 2.19 & 1,52 & $-9.3810^{-6}$ & -3.84 \\
& 139 & 156 & 149 & 1.57 & 1.42 & $-3.2010^{-5}$ & -27.97 \\
& 169 & 155 & 189 & 1.75 & 1.46 & $-2.4310^{-5}$ & -18.16 \\
& 202 & 168 & 213 & 2.19 & 1.52 & $-9.2010^{-6}$ & -3.72 \\
\hline
\end{tabular}

The theoretical temperature sensitivities with the measured dimensions are more negative than the values with the designed dimensions. This result was expected, since the fabricated slot-waveguides have wider slots than the designed values, and thus, the optical power propagating in water is increased. However, it is easy to see the trend to positive values for all the asymmetries, allowing the possibility to approach an athermal slot-waveguide by optimizing the waveguide dimensions. Furthermore, since the measured values of the rails underestimate the widths (the side wall slope of the rails was ignored), we might still have both positive and negatives values when operating in water. 


\section{CONCLUSIONS}

We have demonstrated a method to minimize the temperature sensitivity of slot-waveguides for biosensing applications and the design and fabrication of a Mach-Zehnder interferometer test circuit to evaluate the temperature sensitivity.

The SOI devices were successfully fabricated by using a single lithography step. A first temperature sensitivity and a group index evaluation was made using refractive index matching oil as top cladding. The obtained values are from -70 to $-160 \mathrm{pm} / \mathrm{K}$ and from 1.9 to 2.8 respectively. They confirm the thermal compensation. The TOC values will be close to the athermal point when using water as top cladding. The lowest projected values in this case is $-2 \mathrm{pm} / \mathrm{K}$.

\section{ACKNOWLEDGMENT}

This work was partially supported by the Swedish Research Council under grant agreement B0460801.

\section{REFERENCES}

[1] Tanobe, H., Kondo, Y., Kadota, Y., Okamoto, K. and Yoshikuni, Y., "Temperature insensitive arrayed waveguide gratings on InP substrates," IEEE Photonics Technology Letters 10(2), 235-237 (1998).

[2] Kokubun, Y., Yoneda, S. and Matsuura, S., "Temperature-independent optical filter at $1.55 \mu \mathrm{m}$ wavelength using a silica-based athermal waveguide," Electronics Letters 34(4), 367-369 (1998).

[3] Maru K. and Abe, Y., "Low-loss, flat-passband and athermal arrayed-waveguide grating multi/demultiplexer," Opt. Express 15(26), 18351-18356 (2007).

[4] Claes, T., Molera, J.G., De Vos, K., Schacht, E., Baets, R. and Bienstman, P., "Label-Free Biosensing With a SlotWaveguide-Based Ring Resonator in Silicon on Insulator," IEEE Photonics Journal 1(3), 197-204 (2009).

[5] Almeida, V. R., Xu, Q., Barrios, C. A. and Lipson, M., "Guiding and confining light in void nanostructure.” Opt. Lett. 29(11), 1209-1211 (2004).

[6] Xu, Q., Almeida, V. R., Panepucci, R. R. and Lipson, M., "Experimental demonstration of guiding and confining light in nanometer-size low-refractive-index material.” Opt. Lett. 29(14), 1626-1628 (2004).

[7] Lee, J.-M., Kim, D.-J., Kim, G.-H, Kwon, O.-K., Kim, K.-J. and Kim, G., "Controlling temperature dependence of silicon waveguide using slot structure," Optics Express 16(3), 1645-1652 (2008).

[8] Wang, X., Xiao, S., Zheng, W., Wang, F., Li, Y., Hao, Y., Jiang, X., Wang, M. and Yang, J., "Athermal silicon arrayed waveguide grating with polymer-filled slot structure," Optics Communications 282(14), 2841-2844 (2009).

[9] Zhou, L., Kashiwagi, K., Okamoto, K., Scott, R., Fontaine, N., Ding, D., Akella, V. and Yoo, S., "Towards athermal optically-interconnected computing system using slotted silicon microring resonators and RF-photonic comb generation," Applied Physics A: Materials Science \& Processing 95(4), 1101-1109 (2009).

[10] Zhou, L., Okamoto, K., and Yoo, S. J. B., "Athermalizing and Trimming of Slotted Silicon Microring Resonators With UV-Sensitive PMMA Upper-Cladding," IEEE Photonics Technology Letters 21(17), 1175-1177 (2009).

[11] Huang, H., Ho, S.-T., Huang, D., Tu, Y., Hu, H., Wang, J., and Liu, W., "Slot-waveguide assisted temperatureindependent Mach-Zehnder interferometer based optical filter," Journal of Modern Optics 57(7), 545-551 (2010).

[12] Gylfason, K. B., Carlborg, C. F., Kazmierczak, A., Dortu, F., Sohlström, H., Vivien, L., Barrios, C. A., van der Wijngaart, W., and Stemme, G., "On-chip temperature compensation in an integrated slot-waveguide ring resonator refractive index sensor array," Opt. Express, 18(4), 3226-3237, (2010).

[13] Atsumi, Y., Inoue, K., Nishiyama, N. and Arai, S., "Athermal Wavelength Characteristics of Si Slot Ring Resonator Embedded with Benzocyclobutene for Optoelectronic Integrated Circuits," Japanese Journal of Applied Physics 49(5), 50206-50209 (2010).

[14] Harvey, A. H., Gallagher, J. S., and Sengers, J. M. H. L., "Revised Formulation for the Refractive Index of Water and Steam as a Function of Wavelength Temperature and Density," Journal of Physical and Chemical Reference Data 27(4), 761-774 (1998).

[15] Eldada, L., "Advances in telecom and datacom optical components," Optical Engineering 40(7), 1165-1178 (2001).

[16] IMEC Standard SiPhotonics, http://www.epixfab.eu/index.php?option=com_content\&view=article\&id=49\&Itemid=61, (2012) 
[17] Hammer, M., Hiremath, K. R. and Stoffer, R., "Analytical approaches to the description of optical microresonator devices," AIP Conf. Proc. 709(1), 48-71 (2004).

[18] Baehr-Jones, T., Hochberg, M., Walker, C., and Scherer, A., "High-Q optical resonators in silicon-on-insulatorbased slot waveguides," Applied Physics Letters 86(8), 081101+ (2005).

[19] Heaton, J. M., Jenkins, R. M., Wight, D. R., Parker, J. T., Birbeck, J. C. H., and Hilton, K. P., "Novel 1-to-N way integrated optical beam splitters using symmetric mode mixing in GaAs/AlGaAs multimode waveguides," Applied Physics Letters 61(15), 1754-1756 (1992).

[20] Izutsu, M., Nakai, Y., and Sueta, T., "Operation mechanism of the single-mode optical-waveguide Y junction," Opt. Lett. 7(3), 136-138 (1982).

[21] Quan, Y., Han, P., Ran, Q., Zeng, F,. Gao, L., and Zhao, C., "A photonic wire-based directional coupler based on SOI," Optics Communications 281(11), 3105-3110 (2008).

[22] Llobera Adan, A., [Integrated Optics Technology on Silicon: Optical Transducers], Ph.D. dissertation Universitat Autònoma de Barcelona, Barcelona, (2002).

[23] Seyringer, D., "Low loss and high uniformity multimode interference splitter operating in a wide wavelength band," 9th International Conference on Optical Communications and Networks, 383-386 (2010).

[24] Darmawan, S., Lee, S. Y., Lee, C. W. and Chin, M. K., "Transition and comparison between directional couplers and multimode interferometer based on ridge waveguides," Proc. SPIE 5644, 52-64 (2005).

[25] Blasco J. and Barrios, C. A., "Compact slot-waveguide/channel-waveguide mode-converter," Conference on Lasers and Electro-Optics Europe, 607-607 (2005).

[26] Wang, Z., Zhu, N., Tang, Y., Wosinski, L., Dai, D., and He, S., "Ultracompact low-loss coupler between strip and slot waveguides," Opt. Lett. 34(10), 1498-1500 (2009).

[27] Feng, N.-N., Sun, R., Kimerling, L. C., and Michel, J., "Lossless strip-to-slot waveguide transformer," Opt. Lett. 32(10), 1250-1252 (2007).

[28] Frish, M., Fijol, J., Fike, E. E., Jacobson, S. A., Keating, P. B., Kessler, W. J., LeBlanc, J., Bozler, C., and Fritze, M., "Coupling of single mode fibers to planar Si waveguides using vertically tapered mode converters," OSA Trends in Optics and Photonics 78, (2002).

[29] Wang, Q., Loh, T.-H., Ng, D. K. T. and Ho, S.-T., "Design and Analysis of Optical Coupling Between Silicon Nanophotonic Waveguide and Standard Single-Mode Fiber Using an Integrated Asymmetric Super-GRIN Lens," IEEE Journal of Selected Topics in Quantum Electronics 17(3), 581-589 (2011).

[30] Thorlabs, Single Mode Fiber 1260 to $1620 \mathrm{~nm}$ SMF-28-J9, (2011), http://www.thorlabs.de/thorcat/2200/2292-S01.pdf

[31] Taillaert, D., Van Laere, F., Ayre, M., Bogaerts, W., Van Thourhout, D., Bienstman, P., and Baets, R., "Grating Couplers for Coupling between Optical Fibers and Nanophotonic Waveguides," Japanese Journal of Applied Physics 45(8A), 6071-6077 (2006).

[32] Liu, L., Pu, M., Yvind, K., and Hvam, J. M., "High-efficiency, large-bandwidth silicon-on-insulator grating coupler based on a fully-etched photonic crystal structure," Applied Physics Letters 96(5), 051126+ (2010).

[33] Selvaraja, S. K., Vermeulen, D., Schaekers, M., Sleeckx, E., Bogaerts, W., Roelkens, G., Dumon, P., Van Thourhout, D., and Baets, R., "Highly Efficient Grating Coupler between Optical Fiber and Silicon Photonic Circuit," Conference on Lasers and Electro-Optics/International Quantum Electronics Conference - OSA, CTuC6+ (2009).

[34] Antelius, M., Gylfason, K. B., and Sohlström, H., “An apodized SOI waveguide-to-fiber surface grating coupler for single lithography silicon photonics," Opt. Express 19(4), 3592-3598 (2011).

[35] Samii Y. R., and Michielssen, E., [Electromagnetic Optimization by Genetic Algorithms], John Wiley \& Sons Inc New York, (1999).

[36] Dow Corning® XR-1541 E-Beam resist, Hydrogen silsesquioxane electron beam spin-on resist, http://www.dowcorning.com/applications/search/products/default.aspx? R=8812EN\&\#38; country=GBR

[37] van de Kraats A., and Murali, R., "Proximity Effect in E-beam Lithography," http://nanolithography.gatech.edu/proximity.pdf

[38] Seo, E. Choi., B. and Kim, O., "Determination of proximity effect parameters and the shape bias parameter in electron beam lithography," Microelectronic Engineering 53(1-4), 305-308 (2000).

[39] I. Cargille Laboratories, Fused silica matching liquid, (2002). http://www.cargille.com/FS50350.pdf 